\title{
Hybrid inflation with moduli stabilization and low scale supersymmetry breaking
}

\author{
Sander Mooij and Marieke Postma \\ NIKHEF, Science Park 105, 1098 XG Amsterdam, The Netherlands. \\ smooij@nikhef.nl,mpostma@nikhef.nl
}

\begin{abstract}
We study the supergravity hybrid inflation model of Ref. [1] in the presence of a modulus field. The $\eta$-problem is solved by a shift symmetry for the inflaton, which protects the inflaton mass even in the presence of the modulus field. Inflation is (nearly) unaffected by moduli stabilization, provided the scale of supersymmetry breaking in the post-inflation vacuum is small. Therefore the model has the nice phenomenology that it combines low scale supersymmetry breaking with high scale (grand unification scale) inflation.
\end{abstract}




\section{Contents}

1. Introduction 1

2. The model: SUGRA hybrid inflation 3

3. Adding the modulus sector 5

3.1 General approach 6

3.2 Discussion 9

4. Inflation with a KL modulus sector

4.1 Inflation 10

4.2 Numerical analysis

5. Conclusions 14

A. Coleman-Weinberg potential

\section{Introduction}

Inflation, a short period of rapid expansion in the very early universe, is by now regarded as a fundamental part of standard cosmology, as it solves the horizon problem, the flatness problem and the monopole problem in one go. Furthermore the mechanism of hybrid inflation [2], inflation that naturally comes to an end when a field different from the inflaton field becomes tachyonic, has been shown to have a natural embedding in the framework of supersymmetry. All couplings can be chosen to be of order one (no naturalness problem) and the field direction corresponding to the inflaton can easily be flat enough for slow-roll inflation (no $\eta$-problem). When moving on to local supersymmetry (supergravity) [3], the $\eta$-problem may be circumvented by invoking a shift symmetry in the Kähler potential, as was first proposed in 迎, 刨.

It is well known that inflation is a UV sensitive theory; indeed, this is the root of the $\eta$-problem [6, 7]. In the language of effective field theory this can be readily seen: higher order operators that are suppressed by some large cutoff scale can nevertheless give a large and dominant contribution to the inflaton mass, and thus to the $\eta$-parameter. To fully study inflation it is therefore imperative to consider a UV completion of the theory. In this paper we consider embedding inflation in a higher dimensional Planck-scale theory, for example string theory. After dimensional reduction, the $4 \mathrm{D}$ effective field theory will still carry traces of its 
higher dimensional origin in the form of moduli fields, light scalar fields which parametrize the shapes and sizes of the compactified extra dimensional manifold. For definiteness, we will follow the seminal work of KKLT [8] and assume that all moduli can be fixed at some high scale by fluxes, except for the volume modulus which is to be stabilized at a lower scale by non-perturbative effects. The dynamics of the the volume modulus thus enters the low energy effective field theory, and inflation should be studied in conjunction with modulus stabilization.

There are two ways to deal with the moduli fields in the context of inflation. The first is to make the moduli part of the inflaton dynamics. This is for example done in racetrack [9, 10] and Kähler [11, 12] inflation models, where a modulus field is identified with the inflaton field itself. Another approach, the one we follow in this paper, is to decouple the physics of moduli stabilization from the inflationary physics as much as possible. Our set-up is as follows: we have a hybrid inflation sector and a (volume) modulus stabilization sector, which are coupled only gravitationally as dictated by the SUGRA action. Even though gravitational interactions are usually thought of as being weak, they are generically strong enough to ruin inflation inflation is UV sensitive. It has indeed been shown that "standard" SUSY hybrid inflation [13] cannot be combined with a KKLT-like modulus sector [14, 15] (but see [16] for a possible resolution). Instead we will consider a modified model of hybrid inflation [1].

We want to extend the hybrid inflation model of Ref. [1] with a modulus sector. In our set-up the $\eta$-problem is solved by a shift symmetry for the inflaton, and in addition the property that the inflationary superpotential and its first derivative w.r.t. the inflaton field vanishes during inflation. Note in this respect that a shift symmetry alone is not enough, as it is broken explicitly by the superpotential. This is what goes wrong in standard hybrid inflation. However, the $\eta$-problem is not the only potential difficulty. Making sure that the modulus field remains stabilized during inflation, implies that the scales appearing in the SUSY breaking modulus sector are large, resulting in a large gravitino mass. Even though the inflaton direction is protected, large soft corrections may destabilize the inflationary trajectory.

In this paper we describe an explicit way to stabilize the modulus sector without running into the aforementioned troubles. The trick is to constrain the modulus sector in such a way that its gravitino mass is much smaller than the other scales in the problem. Although this presents some amount of tuning, the result is a phenomenologically favored scenario with low scale SUSY breaking and high scale inflation. An explicit modulus sector that does the job is the model developed by Kallosh \& Linde [17, 18] (we will refer to this as the KL model).

Many SUGRA or string-derived models of inflation predict a large gravitino mass. In models based on a generic KKLT potential the gravitino mass has to be larger than the Hubble constant during inflation $m_{3 / 2} \gtrsim H_{*}$ [17, 18], whereas in models with a large volume compactification the bound is even stronger $m_{3 / 2}^{3 / 2} \gtrsim H_{*}$ 19. It has proven hard to avoid this bound. The KL moduli potential decouples the SUSY breaking scale from the modulus mass, at the cost of tuning, thereby invalidating the bound. Although it is not automatic that a 
KL-based inflation scenario with low scale SUSY breaking can be constructed [14, 15, 20], successful models have been found [21], and the model discussed in this paper is another example. Other approaches to obtain a light gravitino can be found in Refs. [16, 19, 22, 23, 24].

This paper is organized as follows. In section 2 we first briefly describe the shift-symmetric super gravitational model of hybrid inflation introduced in Ref. [1]. Then, in section 3, we explain why combining it with a generic KKLT-type modulus sector does not work: it is impossible to find a suitable inflationary trajectory stable in field space. In the fourth section we show that a constrained modulus sector, of which KL is an explicit example, saves inflation. We discuss the inflationary observables, and show some numerical results. The paper concludes with a discussion of our results.

\section{The model: SUGRA hybrid inflation}

We briefly describe the supergravitational shift-symmetric model of hybrid inflation, that we want to extend by including a moduli sector in the next sections. For a more detailed introduction we refer to the original paper: Ref. [1].

The model is defined by its superpotential $W_{\text {inf }}$

$$
W_{\mathrm{inf}}=\kappa S\left(H^{2}-M^{2}\right)+\frac{\lambda}{\Lambda} N^{2} H^{2},
$$

and Kähler potential $K_{\mathrm{inf}}$

$$
\begin{aligned}
K_{\mathrm{inf}} & =|H|^{2}+|S|^{2}+\frac{1}{2}\left(N+N^{*}\right)^{2}+\frac{\kappa_{H}}{\Lambda^{2}}|H|^{4}+\frac{\kappa_{S}}{\Lambda^{2}}|S|^{4}+\frac{\kappa_{N}}{4 \Lambda^{2}}\left(N+N^{*}\right)^{4} \\
& +\frac{\kappa_{S H}}{\Lambda^{2}}|S|^{2}|H|^{2}+\frac{\kappa_{S N}}{2 \Lambda^{2}}|S|^{2}\left(N+N^{*}\right)^{2}+\frac{\kappa_{H N}}{2 \Lambda^{2}}|H|^{2}\left(N+N^{*}\right)^{2}+\ldots
\end{aligned}
$$

where the ellipses denote higher order terms, and $\Lambda$ is some cutoff scale. The superfield $H$ plays the role of waterfall field responsible for ending inflation. The superfield $S$ is the socalled driving field, as its $F$-term provides the energy density that drives inflation. Finally, the imaginary part of $N$ is the slowly rolling inflaton field. It is hoped that $N$ can be identified with the right-handed sneutrino superfield, and $H$ with the grand unified Higgs field that breaks $B$ - $L$, thus providing an embedding of the model in a grand unified theory [25]. The Kähler potential is invariant under a shift of $N \rightarrow N+i \mu$; this is the before mentioned shift symmetry pivotal for keeping the inflaton direction flat. The superfields can be decomposed in real and imaginary components: $H=\left(h_{r}+i h_{i}\right) / \sqrt{2}, S=\left(s_{r}+i s_{i}\right) / \sqrt{2}$ and $N=\left(n_{r}+i n_{i}\right) / \sqrt{2}$.

Inflation In this model inflation takes place as the field $n_{i}$, the imaginary part of $N$, slowly rolls down to a critical value $n_{i}^{c}$, while the other fields are in their minimum $\left(h_{r}, h_{i}, s_{r}, s_{i}, n_{r}\right)=$ $(0,0,0,0,0)$. Let us first check the stability of this minimum and then briefly explain how inflation comes about. 
During inflation the $F$-term scalar potential ${ }^{1}$

$$
V_{F}=\mathrm{e}^{K}\left[D_{i} W K^{i \bar{j}} D_{\bar{j}} \bar{W}-3|W|^{2}\right],
$$

with $D_{i} W=W_{i}+K_{i} W$ is constant:

$$
V_{\text {tree }}=\kappa^{2} M^{4},
$$

and drives inflation with $H_{\mathrm{inf}}^{2}=V_{\text {tree }} / 3$. All non-inflationary fields are at an extremum of the potential independent of the value of $n_{i}$. Hence the inflationary valley is a classically and quantum mechanically stable trajectory provided all masses squared are positive, and the mass exceeds the Hubble scale during inflation. The masses during inflation are

$$
\begin{aligned}
& \left\{m_{h_{r}}^{2}, m_{h_{i}}^{2}\right\}=\left\{M^{2} \kappa^{2}\left(M^{2}\left(1-\kappa_{S H}\right)-2\right)+\lambda^{2} n_{i}^{4}, M^{2} \kappa^{2}\left(M^{2}\left(1-\kappa_{S H}\right)+2\right)+\lambda^{2} n_{i}^{4}\right\}, \\
& \left\{m_{s_{r}}^{2}, m_{s_{i}}^{2}\right\}=\left\{-4 M^{4} \kappa^{2} \kappa_{S},-4 M^{4} \kappa^{2} \kappa_{S}\right\}, \\
& \left\{m_{n_{r}}^{2}, m_{n_{i}}^{2}\right\}=\left\{2 M^{4} \kappa^{2}\left(1-\kappa_{S N}\right), 0\right\} .
\end{aligned}
$$

Here, and from now on, we set $\Lambda=1$. We see that $h_{r}$ becomes tachyonic when $n_{i}$ drops below the critical value $\left(n_{i}^{2}\right)^{c} \approx \sqrt{2} \kappa M / \lambda$. This will mark the end of inflation. The $s_{r}, s_{i}$ and $n_{r}$ directions are stable as long as $\kappa_{S N}<\frac{5}{6}$ and $\kappa_{S}<-\frac{1}{12}$. This is one of the reasons for including the higher order terms in the Kähler potential (2.2). (The other is that the inflationary observables depend on $\kappa_{S H}$ [1; taking $\kappa_{S H}=\mathcal{O}(10)$ the spectral index can be brought closer to the WMAP central value.)

Thanks to the shift symmetry, $n_{i}$ itself does not acquire any mass, independent of the higher order terms in the Kähler potential; at tree level it is a flat direction in field space. The slow roll parameter $\eta=V^{\prime \prime} / V$ is small, with prime denoting derivative w.r.t. the canonically normalized inflation field, and there is no $\eta$-problem. This is in contrast with "standard" SUSY hybrid inflation 13] where higher order terms lift the flatness of the potential, and thus must be tuned [3]. The reason for this marked difference is that in our model $W_{\text {inf }}$ vanishes during inflation (as well as many first and second derivatives of $W_{\mathrm{inf}}$ ), thereby killing all possible inflaton mass terms. In standard SUSY hybrid inflation on the other hand $W_{\text {inf }} \neq 0$, and the $\eta$-problem resurfaces despite the shift symmetry. It is this remarkable property of the inflaton superpotential that led the authors of [1] to suggest that the model can be combined with a modulus sector. In the next sections we will take a closer look at this claim.

The inflaton potential is generated by the 1-loop Coleman-Weinberg potential [26], from the mass splitting between fermions and bosons. Only the waterfall fields have inflatondependent mass terms and contribute to the inflaton potential. Writing the mass of the waterfall fields and their fermionic superpartners in the form $m_{h_{r, i}}^{2}=\mu^{2}\left(x^{2}+y^{2} \pm 1\right)$ and $\tilde{m}_{h_{r, i}}^{2}=\mu^{2} x^{2}$ with

$$
\mu^{2}=2 \kappa^{2} M^{2}, \quad x=\frac{\lambda^{2} n_{i}^{4}}{2 \kappa^{2} M^{2}}, \quad y=\frac{M^{2}}{2}\left(1-\kappa_{S H}\right),
$$

\footnotetext{
${ }^{1}$ We set the reduced Plank mass $m_{\mathrm{p}}=\left(8 \pi G_{N}\right)^{-1 / 2}=1$.
} 
then the loop potential is given by (A.4) in appendix A. We now have the effective potential

$$
V_{\text {inf }}=V_{\text {tree }}+V_{\text {loop }}\left(n_{i}\right)
$$

The $n_{i}$-direction in field space, flat at tree-level, gets slightly lifted at the one-loop level. With a suitable choice of parameters this effective potential can generate inflation. The inflaton field $n_{i}$ slowly rolls down until it reaches the critical value $n_{i}^{c}$ where inflation ends. We note that for $y^{2}<0$, or equivalently $\kappa_{S H}>1$, the $\mathrm{CW}$-potential has a maximum at $n_{i}^{\max }$ given in (A.6). This introduces a constraint on the initial field value of the inflaton field which has to be smaller than $n_{i}^{\max }$, to make sure that the inflaton rolls towards the "right" minimum. On the other hand for $y^{2}<0$, the loop potential steadily increases with $n_{i}$, and there is no such problem.

With the potential (2.7) one can calculate the inflaton value $\left(n_{i}\right)_{*}$ at horizon-exit, 60 efolds before the end of inflation, when observable scales leave the horizon. Here the slow-roll parameters $\epsilon, \eta$ and $\xi^{2}$ can be evaluated, and consequently the power spectrum $\mathcal{P}_{\mathcal{R}}$, the scalar spectral index $n_{S}$, the tensor-to-scalar ratio $r$, and the running of the scalar spectral index $d n_{S} / d \log k$.

After inflation When the inflation field $n_{i}$ reaches its critical value $n_{i}^{c}$, the waterfall field $h_{r}$ becomes tachyonic. Inflation ends with a phase transition during which the waterfall field obtains a non-zero vev. The post-inflationary vacuum field values are $\left\{h_{r}, h_{i}, s_{r}, s_{i}, n_{r}, n_{i}\right\}=$ $\{ \pm \sqrt{2} M, 0,0,0,0,0\}$, and $V=0$ corresponding to zero cosmological constant.

Numerical results In Ref. [1] it is shown that in the parameter space

$$
\left(\kappa=\mathcal{O}\left(10^{-1}\right), M=\mathcal{O}\left(10^{-3}\right), \lambda=\mathcal{O}\left(10^{-1}\right), \kappa_{S H}=\mathcal{O}(1-10)\right)
$$

many solutions can be found that satisfy the WMAP $1 \sigma$ range for the power spectrum $\mathcal{P}_{\mathcal{R}}^{\frac{1}{2}}=$ $(5.0 \pm 0.1) \times 10^{-5}$ and scalar spectral index $n_{s}=0.960_{-0.013}^{+0.014}$ [27]. The tensor to scalar ratio $r$ typically becomes of order $\left(10^{-5}\right)$ which easily satisfies the WMAP bound $r<0.2$. The model fails on the prediction of $d n_{s} / d \log k$ : it typically predicts a value of order $\left(10^{-4}\right)$ while

WMAP measured $-0.0032_{-0.020}^{+0.021}$. As the accuracy of this measurement is rather low, this does not seem to be a serious problem.

\section{Adding the modulus sector}

The inflaton model described in the previous section is an effective theory, arising as a lowenergy effective description of an underlying Planck scale theory. If the UV completion is an extra dimensional theory, we expect moduli fields to appear in the $4 \mathrm{D}$ effective action. The moduli fields parametrize the sizes and shapes of the extra dimensions. In case the vacuum manifold is degenerate, the moduli correspond to massless modes appearing in the low energy effective four-dimensional theory. 
For definiteness we concentrate in this paper on a KKLT type moduli sector [8], arising from compactifications in type IIB string theory. KKLT showed that all complex structure moduli (shape moduli) can be stabilized by fluxes. In the simplest case there is only one Kähler modulus (size modulus) left, the volume modulus, which appears in the $4 \mathrm{D}$ effective theory. This modulus, in turn, is stabilized by invoking non-perturbative effects, coming from either gaugino condensation or instantons. Finally, to arrive at a zero cosmological constant, a non-supersymmetric uplifting term is added, generated by an anti-D3 brane located at the bottom of a throat in the compactification manifold.

To combine the inflaton and modulus sector we simply add their respective Kähler- and superpotentials $^{2}$ :

$$
W=W_{\mathrm{inf}}+W_{\mathrm{mod}}, \quad K=K_{\mathrm{inf}}+K_{\mathrm{mod}}
$$

with

$$
K_{\text {mod }}=-3 \ln (T+\bar{T})
$$

For the moment we ignore corrections to this tree level Kähler potential. These will be treated in section 4.1 .

\subsection{General approach}

The function $W_{\text {mod }}(T)$ generically contains a constant term $W_{0}$ arising from integrating out the stabilized moduli and a non-perturbative potential that is to stabilize $T$. In this section we will work with a generic function $W_{\text {mod }}(T)$ and see what restrictions on this function we get to make inflation work in this moduli-extended framework. We choose a KKLT uplifting potential $V_{\text {up }}=c /(T+\bar{T})^{2}$, with $c$ a constant tuned to solve the cosmological constant problem. However, its specific form is not so important for our discussion.

As before the modulus fields can be decomposed in real and imaginary parts: $T=\sigma+i \alpha$. Choosing the phases in the superpotential judiciously, we can set $\alpha=0$ to zero consistently. We define $\sigma=\sigma_{0}$ at the minimum of the F-term modulus potential in the absence of the inflaton sector, i.e.

$$
\left.\partial_{\sigma} V_{\bmod }^{F}\right|_{\sigma=\sigma_{0}}=\left.0 \quad \Leftrightarrow \quad D_{T} W\right|_{\sigma=\sigma_{0}}=0
$$

Due to the uplift term and the presence of the inflaton sector, $\sigma$ is displaced from its F-term minimum both during and after inflation. If the displacement is minimal the inflationary trajectory and the post-inflation minimum are only slightly affected as well, and the moduli sector may be combined with inflation. In this case $\sigma \approx \sigma_{0}$ and $D_{T} W \approx 0$ are still good approximations. In the rest of this section we discuss the general conditions the moduli sector has to satisfy for this to be the case, followed - in the next section — by an explicit example. There are many pitfalls. When a modulus sector is included, the $\eta$-problem may reappear, the vacuum after inflation and/or the inflationary trajectory may be destabilized, and the corrections to the waterfall fields may hamper a successful exit to inflation.

\footnotetext{
${ }^{2}$ The Kähler potential does not have to be separable in modulus and inflaton field, e.g. $K_{\text {inf }}$ can appear inside the log. We checked that its exact form does not affect our qualitative results.
} 
$\eta$-problem We have seen that in the absence of a moduli sector the tree level inflaton mass is zero, as a consequence of the shift symmetry and the fact that $W_{\mathrm{inf}}=0$. Due to the shift symmetry the Kähler potential is independent of $n_{i}$, and thus any mass for $n_{i}$ must come from the second derivative of the term in square brackets in (2.3). The fact that the modulus superpotential is non-zero does not change the results, the inflaton potential is still flat at tree level. All terms in $m_{n_{i}}^{2}$ proportional to $W_{\text {mod }}$ or its derivatives are multiplied by $\left(W_{\text {inf }}\right)_{n_{i} n_{i}}$, which is zero during inflation. As the $\eta$-problem is usually the main obstacle to embedding inflation in a supergravity theory, this is no small feat.

Stability of the vacuum Consider the vacuum after inflation. We suppose the postinflationary minimum to occur at $\left\{h_{r}, h_{i}, s_{r}, s_{i}, n_{r}, n_{i}, \sigma, \alpha\right\}=\left\{ \pm \sqrt{2} M, 0,0,0,0,0, \sigma_{0}, 0\right\}$. For the post-inflation scalar potential we find

$$
V_{\mathrm{vac}}=\frac{c}{(4 \sigma)^{2}}+V_{\mathrm{mod}}^{F}+f\left(M^{2}\right), \quad V_{\mathrm{mod}}^{F}=\frac{-3 W_{\mathrm{mod}}^{2}+\frac{4}{3} \sigma^{2}\left(D_{T} W_{\mathrm{mod}}\right)^{2}}{(2 \sigma)^{3}}
$$

where each term in $f\left(M^{2}\right)$ is either proportional to $D_{T} W$ or to $W$ (with $D_{T} W=-3 W /(2 \sigma)+$ $W_{T}$ ). For parameters that keep the modulus stabilized during inflation (discussed below), the $M$-dependent corrections $f\left(M^{2}\right)$ to the modulus potential after inflation are small, and do not destabilize the potential minimum.

The first derivatives of the scalar potential with respect to the eight real fields, evaluated at the postulated potential minimum after inflation, are manifestly zero or involve again small functions of $M^{2}$ proportional to $D_{T} W$ or $W$ indicating that the minimum of some of the fields is slightly displaced. One of the displaced fields is the modulus field, which is shifted from its F-term potential minimum $\sigma_{0}$ due to the presence of the uplift term. This shift is typically small.

Second derivatives again involve many functions of $D_{T} W, W$ and $c$. The vacuum mass of the field $n_{r}$ is most seriously affected by moduli corrections, and runs the risk of going tachyonic:

$$
\left.m_{n_{R}}^{2}\right|_{\mathrm{vac}}=\frac{4\left(D_{T} W_{\mathrm{mod}}\right)^{2} \sigma^{2}-3 W_{\mathrm{mod}}^{2}+\mathcal{O}\left(M^{2}\right)}{12 \sigma^{3}} .
$$

Indeed, for $D_{T} W_{\text {mod }} \approx 0$ the mass is tachyonic unless $W_{\text {mod }} \lesssim M$ is sufficiently small, and the $\mathcal{O}\left(M^{2}\right)$ terms dominate.

Stability during inflation The tentative inflationary trajectory is

$$
\left\{h_{r}, h_{i}, s_{r}, s_{i}, n_{r}, n_{i}, \sigma, \alpha\right\}=\left\{0,0,0,0,0, n_{i}, \sigma_{0}, 0\right\}
$$

We have to check whether this is still an extremum when the modulus potential is turned on. As before $n_{i}$ is the slowly rolling inflaton field. The potential during inflation along this trajectory is then

$$
V_{\mathrm{inf}}=\frac{c}{(4 \sigma)^{2}}+V_{\mathrm{mod}}^{F}+\frac{\kappa^{2} M^{4}}{(2 \sigma)^{3}}
$$


with $V_{\text {mod }}^{F}$ defined in (3.4). If $\sigma \approx \sigma_{0}$ the first two terms in the above expression nearly cancel, and the last term is as before the energy density driving inflation. However, this energy density is now modulus dependent. If this term is too large, the displacement in $\sigma$ is large, or worse, the barrier separating in the potential disappears and $\sigma$ rolls off to infinity.

The fields are all at an extremum for the inflationary trajectory, except for the modulus and the $s_{r}$ field. The non-vanishing first derivatives are

$$
\begin{aligned}
\partial_{s_{r}} V_{\mathrm{inf}} & =\frac{\kappa M^{2}\left(\left(D_{T} W_{\mathrm{mod}}\right) \sigma+W_{\mathrm{mod}}\right)}{2 \sqrt{2} \sigma^{3}}, \\
\partial_{\sigma} V_{\mathrm{inf}} & =\frac{-3\left(3 \kappa^{2} M^{4}+4 c \sigma\right)+8 \sigma^{2}\left(D_{T} W_{\mathrm{mod}}\right)\left(-2 D_{T} W_{\mathrm{mod}}-\frac{3 W_{\mathrm{mod}}}{\sigma}+\sigma W_{\mathrm{mod}}^{\prime \prime}\right)}{24 \sigma^{4}},
\end{aligned}
$$

where primes denote derivatives with respect to $\sigma$. We see indeed that during inflation the minimum of the $\sigma$-field does not occur at exactly $\sigma=\sigma_{0}$ : now it is both the uplift and the inflationary energy density that shifts the minimum away. In addition, the field $s_{r}$ is not minimized at $s_{r}=0$. For $D_{T} W_{\text {mod }} \approx 0$, the first derivative is proportional to $W_{\text {mod }}$ and is typically large. This can have dramatic consequences, as we will see. The matrix of second derivatives evaluated at the inflationary minimum is not diagonal anymore, as $V_{s_{r} \sigma}$ does not vanish. This coupling between $s_{r}$ and $\sigma$ could already be foreseen from (3.8). We also find a similar coupling between $s_{i}$ and $\alpha$, but as they both have their minimum at zero this coupling will not have any significant consequences.

Just as in the case without moduli fields ( $c f$. the discussion below (2.5)), we need some tuning of the $\kappa$-parameters in the inflationary Kähler potential to maintain positive definite masses squared. Since expressions are long, we only explicitly give the mass of the field $h_{r}$, that can be compared to 2.5)

$$
\begin{aligned}
V_{h_{r} h_{r}}^{\inf }=\frac{1}{\left(2 \sigma_{0}\right)^{3}}[ & \kappa^{2} M^{2}\left(\left(M^{2}\left(1-\kappa_{S H}\right)-2\right)+\lambda^{2} n_{i}^{4}-2 W_{\bmod }^{2}+2 \sigma \lambda n_{i}^{2} D_{T} W_{\text {mod }}\right. \\
& \left.+W_{\bmod }\left(\lambda n_{i}^{2}-4 \sigma D_{T} W_{\text {mod }}\right)+\frac{4}{3} \sigma D_{T} W_{\bmod }\left(\sigma D_{T} W_{\text {mod }}+3\right)\right] .
\end{aligned}
$$

Once again, for $D_{T} W_{\text {mod }} \approx 0$, the corrections - in this case to the waterfall masses - scale with $W_{\text {mod }}$ and are potentially large.

Waterfall mechanism and CW-loop The above expression becomes much more complicated when we take the displacement of $s_{r}$ into account, see (3.8). If we allow $s_{r}$ to be non-zero we find among many other terms

$$
\delta m_{h_{r, i}}^{2}=\frac{\kappa^{2} s_{r}^{2}}{2 \sigma^{3}}+\ldots
$$

This indicates that the shift in $s_{r}$ can do a lot of harm to our model. Once the waterfall masses get dominated by terms like (3.10), the Coleman-Weinberg loop potential changes drastically and inflation is no longer possible. Therefore, we absolutely need the displacement in $s_{r}$ to be small. 


\subsection{Discussion}

As discussed, for a generic superpotential $\left(D_{T} W_{\text {mod }}\right) \approx 0$ as this minimizes the F-term superpotential (3.3), and corrections to the inflaton potential scale with $W_{\text {mod }}$ (which is the only scale in the moduli sector). $W_{\text {mod }}$ should be large enough to assure the modulus remains stabilized during inflation, yet small enough to ensure that the vacuum and inflationary trajectory is not destabilized. This does not seem to be easy. And indeed, for a KKLT modulus sector, which is of the above described generic form, this is impossible. In the original KKLT paper [8] the non-perturbative potential is a single exponent, and the superpotential is

$$
W_{\text {mod }}=-W_{0}+A e^{-a T} \text {. }
$$

where the sign in front of $W_{0}$ is chosen such that the potential is minimized by $\alpha=0$. The minimum of $V_{\mathrm{vac}}$ occurs for $D_{T} W_{\text {mod }} \approx 0$ and $W_{\text {mod }} \sim W_{0}$. Let us go through all modulus corrections for this specific choice of superpotential.

For the $n_{r}$-direction to be stable in the vacuum after inflation, see (3.5), we have to demand $W_{0} \lesssim \kappa M^{2}$. From the perspective of the modulus field, the inflationary energy density $\kappa^{2} M^{4}$ acts as an additional uplift term (3.7). If this term is too large, $\sigma$ is destabilized. To avoid this one needs $\kappa^{2} M^{4} /(2 \sigma)^{3} \lesssim V_{\text {up }}$ or

$$
\kappa M^{2} \lesssim W_{0}
$$

It follows that stabilizing the modulus during inflation plus stabilizing the vacuum are both possible only for a very limited range of parameters: $W_{0} \approx \kappa M^{2}$. But what kills the KKLT model are the corrections it gives to the waterfall fields. As anticipated from (3.8) it follows that both $s_{r}$ and $\sigma$ are displaced considerably during inflation. Numerically we find $s_{r} \sim$

$\mathcal{O}\left(10^{-1}-10^{-2}\right)$ (where we used $V_{\text {inf }}^{1 / 4} \sim M_{\text {GUT }}$ ). The corresponding correction to the waterfall field (3.10) is enormous, hampering a graceful exit to inflation.

How to salvage inflation? Taking a look at the modulus corrections (3.5, 3.7, 3.8, 3.10), we see they all vanish in the limit that both

$$
\left.\left.D_{T} W_{\text {mod }}\right|_{\sigma=\sigma_{0}} \approx 0 \quad \& \quad W_{\bmod }\right|_{\sigma=\sigma_{0}} \approx 0 .
$$

The first condition is assured by minimizing of the F-term potential (3.3), but the second constitutes an extra constraint on the modulus potential which can be satisfied by tuning the parameters in the superpotential. Such a tuning is not possible for the one-exponent KKLT model. Kallosh \& Linde (KL) constructed a modulus sector with two exponents, with the parameters carefully tuned, such that (3.13) is satisfied [17, 18]. We will discuss this model in detail in the next section. The only constraint left is then (3.12), assuring that the modulus remains fixed during inflation.

The fine-tuning required to set $W_{\text {mod }} \approx 0$ is the same tuning that creates a hierarchy between the gravitino and modulus mass with $m_{3 / 2} \ll m_{T}$. Since $H_{*} \gtrsim m_{T}$ (from (3.12)), this tuning allows to have low scale SUSY breaking with high scale inflation — something 
that seems impossible in non-fine tuned models. This was the motivation behind the KL model. Note that since in hybrid inflation $V_{\text {inf }} \sim M_{\mathrm{GUT}}^{4}$, without this tuning, it is impossible to get the phenomenologically favored TeV scale SUSY breaking.

Finally we would like to contrast the results with standard SUSY hybrid inflation 13, 14, 15. In the standard case, the $\eta$-problem reappears once a modulus sector is included; the reason is that in these models the inflaton superpotential is non-zero $W_{\text {inf }} \neq 0$, and many terms mixing the modulus and inflaton sector appear in $V_{F}$. In addition, the waterfall masses get large corrections, just as we found above. Although each of these problems can be solved separately by a fine-tuned condition on the modulus potential, they cannot be solved simultaneously. Since in our case, the $\eta$-problem has dropped off the list, inflation can be rescued by a single tuning.

\section{Inflation with a KL modulus sector}

As discussed in the previous section, hybrid inflation may be combined with a modulus sector provided the latter satisfies (3.13). In this section we work out the details, focusing on the KL modulus sector introduced by Kallosh \& Linde in [17, 18]. Augmenting the KKLT potential by an additional non-perturbative exponential factor, it is possible (by tuning the parameters) to construct a SUSY Minkowski minimum with $D_{T} W=W_{T}=0$. The superpotential is

$$
W_{\text {mod }}=-W_{0}+A \mathrm{e}^{-a T}-B \mathrm{e}^{-b T}
$$

with $W_{0}$ and $\sigma_{0}$ :

$$
W_{0}=w_{0} \equiv A\left(\frac{b B}{a A}\right)^{a /(a-b)}-B\left(\frac{b B}{a A}\right)^{b /(a-b)}, \quad \sigma_{0}=\bar{\sigma}_{0} \equiv \frac{1}{a-b} \ln \left(\frac{a A}{b B}\right) .
$$

So, at the cost of fixing $W_{0}$ and introducing another exponent in the non-perturbative potential, we now explicitly have $D_{T} W=W_{T}=0$, and thus $V_{\bmod }^{F}=0$, in the vacuum after inflation $\left\{h_{r}, h_{i}, s_{r}, s_{i}, n_{r}, n_{i}, \sigma, \alpha\right\}=\left\{ \pm \sqrt{2} M, 0,0,0,0,0, \sigma_{0}, 0\right\}$. No uplift term is needed, and SUSY is unbroken.

We can get a small but non-zero gravitino mass by perturbing the SUSY Minkowski solution

$$
W_{0}=w_{0}+\epsilon_{w}
$$

As long as the perturbation is small enough $D_{T} W_{\text {mod }} \approx 0, W_{\text {mod }} \approx \epsilon_{w}$ and (3.13) is still satisfied. We will determine below how small $\epsilon_{w}$ has to be. With this perturbation the minimum of the F-term potential, located at $\sigma_{0}=\bar{\sigma}_{0}+\mathcal{O}\left(\epsilon_{w}\right)$, is SUSY AdS, and a small uplift $V_{\text {up }} \approx 3 W_{0}^{2} /\left(2 \sigma_{0}\right)^{3}$ is needed to get zero cosmological constant. SUSY is broken in the

process. In this set-up there is a large hierarchy between the gravitino $m_{3 / 2}=\mathrm{e}^{K}|W| \propto \epsilon_{w}$ and modulus mass $m_{\sigma} \propto \sqrt{V_{\sigma \sigma}} \propto W_{0}$.

\subsection{Inflation}

Let us see how inflation works for the hybrid inflation model described in section 2 combined with a KL modulus sector. 
Stability of the vacuum Since the function $f\left(M^{2}\right) \sim \epsilon^{2}$ in (3.4) the inflaton corrections to the modulus minimum after inflation are small. Likewise the modulus correction to the inflaton sector are small. The mass of $n_{r}$ in (3.5) is manifestly positive definite in the vacuum. We checked numerically the stability of the vacuum.

Stability during inflation From (3.7) we see that during inflation we now have

$$
V_{\mathrm{inf}} \approx \frac{\kappa^{2} M^{4}+\mathcal{O}\left(\epsilon_{w}^{2}\right)}{\left(2 \sigma_{0}\right)^{3}}
$$

The inflationary trajectory is slightly shifted from the tentative inflationary trajectory (3.6), as the first derivatives $V_{i}$ with $i=\left\{s_{r}, \sigma\right\}$ are non-zero (3.8). Expanding in small $\epsilon_{w}$ this shift is

$$
\begin{aligned}
\delta \sigma & =-\frac{9 \kappa^{2} M^{4}\left(3-4 \kappa_{S}\right)}{4 a^{2} b^{2} \kappa_{S} W_{0}^{2}}+\epsilon_{w} \frac{3\left(1-2 \kappa_{S}\right)}{4 a b \kappa_{s} W_{0} \sigma_{0}}, \\
\delta s_{r} & =-\frac{9 \kappa M^{2}}{8 \sqrt{2} a b W_{0} \kappa_{S} \sigma_{0}^{2}}-\epsilon_{w} \frac{1}{\sqrt{2} \kappa \kappa_{S} M^{2}} .
\end{aligned}
$$

The shift due to the inflation sector, which is the $\epsilon_{w}$ independent part, is small, and harmless for inflation. The corrections due to the modulus sector scale with $\epsilon_{w}$ and can be larger depending on the size of $\epsilon_{w}$. The mass matrix is nearly diagonal. Except for the $s_{r}$-field, the masses for all the inflaton fields are as before (2.5), up to an overall scaling by $\left(2 \sigma_{0}\right)^{3}$, and up to order $\delta m_{i}^{2}=\mathcal{O}\left(\epsilon_{w}^{2} /\left(2 \sigma_{0}\right)^{3}\right)$ corrections. From the masses of $s_{r}$ and $n_{r}$ one can deduce constraints on $\kappa_{S N}$ and $\kappa_{S}$, just as we did before around 2.5:

$$
m_{n_{r}}^{2}=\frac{2 \kappa^{2} M^{4}\left(1-\kappa_{S N}\right)}{\left(2 \sigma_{0}\right)^{3}}, \quad m_{s_{r}}^{2}=\frac{\kappa^{2} M^{4}\left(3-4 \kappa_{S}\right)}{\left(2 \sigma_{0}\right)^{3}}
$$

lead to the constraints $\kappa_{S N}<\frac{5}{6}$ and $\kappa_{S}<\frac{2}{3}$. It follows that for $\epsilon_{w} \gtrsim \kappa M^{2}$ the moduli corrections dominate, and one of the masses, depending on the choice of $\kappa_{i}$ parameters in the Kähler potential (2.2), may go tachyonic, thereby destroying inflation.

Waterfall mechanism and CW-loop A stronger bound on the value of $\epsilon_{w}$ may be obtained by looking at the waterfall masses. Writing the masses of the bosonic waterfall fields and their superpartners in the form $m^{2}=\mu^{2}\left(x^{2}+y^{2} \pm 1\right)$ and $\tilde{m}^{2}=\mu^{2} x^{2}$, we find

$$
\mu^{2}=\frac{2 \kappa^{2} M^{2}}{\left(2 \sigma_{0}\right)^{3}}+\mathcal{O}\left(\epsilon_{w}\right), \quad x^{2}=\frac{\lambda^{2} n_{i}^{4}}{2 \kappa^{2} M^{2}}+\mathcal{O}\left(\epsilon_{w}\right), \quad y^{2}=\frac{M^{2}}{2}\left(1-\kappa_{S H}\right)-\epsilon_{w}^{2} \frac{\kappa_{S H} \lambda^{2} n_{i}^{4}}{4 \kappa^{2} \kappa_{S}^{2} M^{2}}
$$

where the dominant moduli correction for our purposes is the $\mathcal{O}\left(\epsilon_{w}^{2}\right)$ correction in $y^{2}$. Although $y^{2} \ll x^{2}$, and is an unimportant contribution to the waterfall field mass, it is relevant for the 1-loop potential. As explained in the appendix A, the reason is that the dominant terms cancel between the bosons and the fermions in the Coleman-Weinberg potential (A.1). Indeed, even in the absence of moduli corrections the term $\propto M^{2}\left(1-\kappa_{S H}\right)$ in the boson mass 
causes the loop potential to develop a maximum for $\kappa_{S H}>1$ (A.6). If the $\epsilon_{w}^{2}$-correction in $y^{2}$ dominates, the loop potential steepens for large $n_{i}$. In the case of $\kappa_{S H}>0$, this results in the maximum shifting to smaller values of $n_{i}$, until at some point it becomes impossible to get 60 e-folds of inflation. In the opposite limit $\kappa_{S H}<0$ it results in a larger spectral index, in contradiction with observations. Either way, inflation is ruined if the moduli corrections get too large. Using (4.7) this gives the bound

$$
\epsilon_{w} \lesssim 0.1-0.01 \kappa M^{2}
$$

where the exact value depends on the $\kappa_{i}$ values, and the precise parameters. This estimate is confirmed by our numerical calculation.

Corrections to modulus Kähler potential What remains to address is an analysis of possible corrections to the tree-level Kähler moduli potential introduced in (3.2). Denoting $\alpha^{\prime}$ [28] and $g_{s}$ [29, 30] corrections by $\theta_{1}$ and $\theta_{2}$ respectively, we replace (3.2) by

$$
K_{\text {mod }}=-2 \ln \left[(T+\bar{T})^{3 / 2}+\theta_{1}\right]+\frac{\theta_{2}}{(T+\bar{T})^{3 / 2}} .
$$

In the natural limit $\theta_{1}<(T+\bar{T})^{3 / 2}$ we can expand the logarithm to arrive at

$$
K_{\mathrm{mod}}=-3 \ln (T+\bar{T})+\frac{-2 \theta_{1}+\theta_{2}}{(T+\bar{T})^{3 / 2}} \equiv-3 \ln (T+\bar{T})+\frac{\theta}{(T+\bar{T})^{3 / 2}},
$$

where we have defined $\theta \equiv-2 \theta_{1}+\theta_{2}$.

To check analytically whether these corrections might spoil inflation, we calculate the induced displacement in the minimum of the $s_{r}$ field. Before we have seen that large $s_{r}$ contributions ruin the waterfall mechanism and CW loop effect, so this seems the right quantity to consider. In leading order in $M^{2}$ we find that these contributions are naturally small:

$$
\delta s_{r}=\left.\delta s_{r}\right|_{\theta=0}\left(1+\frac{\theta}{(T+\bar{T})^{3 / 2}}\right) .
$$

This already suggests that Kähler corrections leave the model unaffected. Indeed, this is confirmed by our numerical analysis. As long as $\theta<(T+\bar{T})^{3 / 2}$ all results of the previous sections apply: there is no danger for the Kähler corrections to spoil the model.

\subsection{Numerical analysis}

Adding a modulus sector to inflation, the F-term potential and thus all masses squared are rescaled by a factor $\mathrm{e}^{K}=(2 \sigma)^{-3}$. We can absorb this factor in the parameters of the superpotential via

$$
\bar{\kappa}=\frac{\kappa}{\left(2 \sigma_{0}\right)^{3 / 2}}, \quad \bar{\lambda}=\frac{\lambda}{\left(2 \sigma_{0}\right)^{3 / 2}}, \quad \bar{A}=\frac{A}{\left(2 \sigma_{0}\right)^{3 / 2}}, \quad \bar{B}=\frac{B}{\left(2 \sigma_{0}\right)^{3 / 2}}, \quad \bar{W}_{0}=\frac{W_{0}}{\left(2 \sigma_{0}\right)^{3 / 2}} .
$$



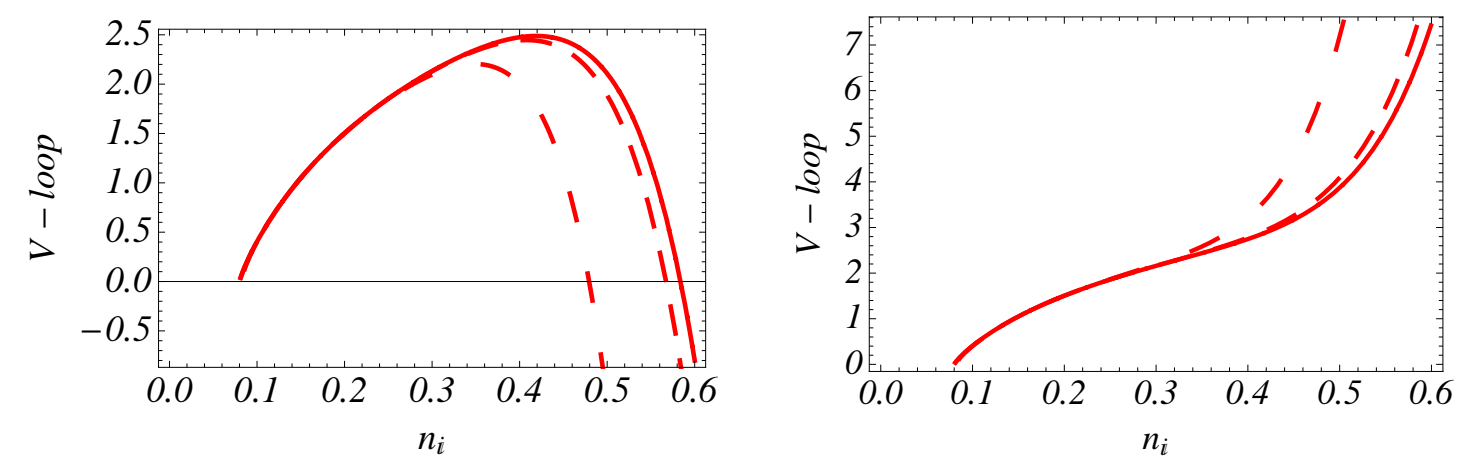

Figure 1: The Coleman-Weinberg potential, rescaled by a factor $10^{15}$, as a function of $n_{i}$ for $\epsilon_{w}=$ $\left\{0,10^{-7}, 10^{-5}\right\}$ corresponding to the solid, short dashed and dashed lines respectively. On the left are the results for $\kappa_{S H}=1$, on the right for $\kappa_{S H}=-1$.

It is the barred quantities that give the effective couplings between the fields, and that can be measured (in principle) in experiments. The rescaling allows to easily compare the parameter space for hybrid inflation without moduli as described in Section 2 and discussed in detail in Ref. [1], with the set-up where a modulus potential is included. If in the former case the model gives the right predictions for the density perturbations for a given set of parameters, for example $\{\kappa=0.14, M=0.003, \ldots\}$, the same observational results are obtained in the setup up with a modulus field if we choose the same numerical values for the barred quantities $\{\bar{\kappa}=0.14, M=0.003, \ldots\}$. This correspondence works up to $\mathcal{O}\left(\epsilon_{w}\right)$ corrections. We checked numerically that with the above identification we get the same parameter space for successful inflation, e.g. including the same $\kappa_{S N}$ dependence, as found in Ref. [1].

Consider an explicit numerical example. For the inflaton sector we choose parameter values

$$
\bar{\kappa}=0.14, \quad M=0.003, \quad \bar{\lambda}=0.1, \quad \kappa_{S H}=1,
$$

and all other $\kappa_{i}$ equal to -1 . As discussed in section 2 this assures stability of the inflationary trajectory. For the modulus sector we take

$$
\bar{A}=1, \quad \bar{B}=1.03, \quad a=\frac{2 \pi}{100}, \quad a=\frac{2 \pi}{99} .
$$

which gives $W_{0}=0.276+\epsilon_{w}$ and $\sigma_{0}=62.41$. The exact parameter values in (4.14) are not so important, what matters is the resultant value for $W_{0}$ and to some lesser extent $\sigma_{0}$.

As anticipated, as we increase $\epsilon_{w}$ we see that the moduli corrections first appear in the loop potential. For $\epsilon_{w}=\left\{0,10^{-7}, 10^{-5}\right\}$ we get $\delta \sigma=\{2.06,2.39,5.33\} \times 10^{-5}$ and $\delta s_{r}=\{3.26,3.66,7.28\} \times 10^{-4}$. These values match our estimates (4.5). Although the increase in $\delta s_{r}$ seems quite moderate, the effects are nevertheless visible in the loop potential, where the maximum is shifting to increasingly small $n_{i}$-values. This is plotted in Fig.1. For $\epsilon=10^{-5}$ the loop potential gets modified in such a way that the slow-roll trajectory is not large enough to accommodate 60 e-folds of inflation. Fig. 11 also shows the equivalent results 

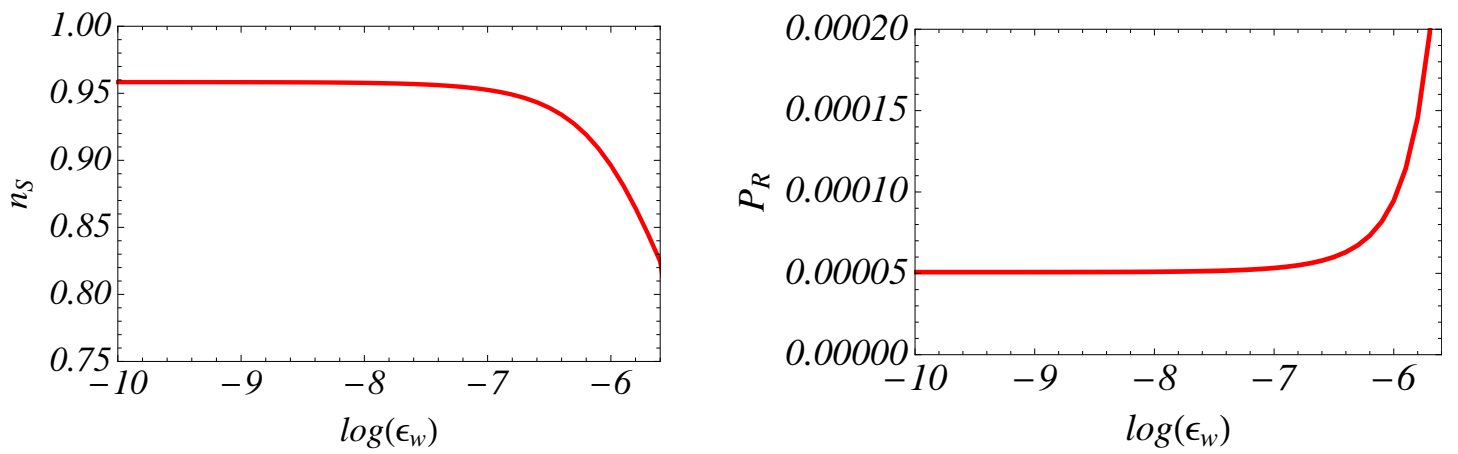

Figure 2: The spectral index $n_{s}$ (left) and power spectrum $P_{\mathcal{R}}$ (right) as a function of $\epsilon_{w}$ for the parameters mentioned in the text.

for $\kappa_{S H}=-1$ and for the rest the same parameters; now the potential steepens to fast for $\epsilon_{w}>10^{-5}$ pushing the spectral index to values larger than one.

Fig. 2 shows the spectral index and power spectrum as a function of $\epsilon_{w}$. For small $\epsilon_{w}$ the results are identical to those found in the model without a modulus. As $\epsilon_{w}$ approaches its critical value (4.8) the results for the spectral index and power spectrum change rapidly, and inflation breaks down abruptly.

\section{Conclusions}

In this paper we combined the hybrid inflation model of [1] with a KKLT like modulus sector. The inflaton mass is protected by a shift symmetry, and remains massless (at tree level) even in the presence of the modulus sector. This is in sharp contrast with standard SUGRA hybrid inflation.

The vacuum after inflation and the inflationary trajectory are corrected by the modulus sector. These corrections are under control and do not disrupt inflation provided the modulus sector satisfies the constraint $D_{T} W_{\text {mod }} \approx W_{\text {mod }} \approx 0$. The first condition is automatic in the minimum of the potential, the second conditions can be satisfied by fine-tuning the parameters in the potential. This is the same fine-tuning needed to get a hierarchy between the gravitino and modulus mass, and which allows for low scale SUSY breaking yet high scale inflation. As explicit examples, the original KKLT modulus stabilization scheme [8] does not satisfy the above condition, whereas the fine-tuned model of Kallosh \& Linde [17, 18] does.

Why inflation works for a modulus sector with small scale SUSY breaking can be easily understood by considering the relevant scales in the system.

1. The modulus mass $m_{T} \propto W_{0}$ which sets the height of the barrier in the modulus potential. It has to be larger than the inflationary scale for the modulus to be stabilized during inflation; this implies the condition (3.12). 
2. The energy density during inflation $V_{\text {inf }}=\bar{\kappa}^{2} M^{4}$, which determines the size of the density perturbations. To get the observed amplitude we find $V_{\text {inf }}^{1 / 4} \sim M_{\mathrm{GUT}}$ is of the order of the grand unified scale.

3. The vacuum gravitino mass $m_{3 / 2}=\mathrm{e}^{K / 2}|W| \propto \epsilon_{W}$, which sets the scale of the moduli corrections to the inflationary potential. It cannot be too large, the bound (4.8) translates in a bound on the gravitino mass $m_{3 / 2} \lesssim 10^{9}-10^{10} \mathrm{GeV} / \sigma_{0}^{3 / 2}$.

In summary, we find that it is possible to extend the, in itself already very promising, model of supersymmetric hybrid inflation proposed in Ref. [1] with a moduli sector. It is absolutely necessary to have a modulus sector that does not break SUSY too badly. Therefore we need to tune the parameters in the superpotential. As a bonus, however, we find that our extended model can accommodate TeV-scale SUSY breaking.

\section{Acknowledgments}

The authors are supported by a VIDI grant from the Dutch Science Organization (NWO).

\section{A. Coleman-Weinberg potential}

The Coleman-Weinberg potential is 26

$$
V_{\mathrm{CW}}=\frac{1}{64 \pi^{2}} \sum_{i}(-1)^{F} m_{i}^{4} \ln \left(\frac{m_{i}^{2}}{\Lambda^{2}}\right)
$$

where the sum is over all masses, with $F=1$ for bosons and $F=-1$ for fermions, and $\Lambda$ is the cutoff scale. Only the $n_{i}$-dependent mass terms lift the inflaton potential, in our case these are the waterfall field masses and their fermionic partners. They can be written in the form

$$
m_{h_{r, i}}^{2}=\mu\left(x^{2}+y^{2} \pm 1\right), \quad \tilde{m}_{h_{r, i}}^{2}=\mu^{2} x^{2}
$$

with $\mu, x, y$ given for inflation without and with a modulus field respectively by (2.6.4.7). The waterfall field $h_{r}$ becomes tachyonic and inflation ends for $x^{c}=1$.

Even though $y^{2} \ll x^{2}$ is clearly subdominant in the expression for the mass terms (A.2), they are important for the shape of the loop potential. This is because the dominant contributions of the boson mass cancels with that of the fermion mass in (A.1). The loop potential becomes

$$
\begin{aligned}
& V_{\mathrm{CW}}=\left(\frac{\mu^{4}}{32 \pi^{2}}\right) {\left[2\left(1+x^{2} y^{2}+y^{4}\right) \ln \left(\frac{x^{2} \mu^{2}}{Q^{2}}\right)\right.} \\
&\left.+\left(x^{2}+y^{2}+1\right)^{2} \ln \left(1+\frac{y^{2}+1}{x^{2}}\right)+\left(x^{2}+y^{2}-1\right)^{2} \ln \left(1+\frac{y^{2}-1}{x^{2}}\right)\right],
\end{aligned}
$$

with $Q$ the renormalization scale which we fix to $Q=\mu=\left.\tilde{m}_{h_{r, i}}\right|_{x=1}$. For negative values $y^{2}<0$ the potential develops a maximum at large $x$. Inflation has to take place on the left 
of the maximum, for the inflaton field to roll towards the "right" minimum. This also means that if the maximum is to close to the critical value, it is impossible to get 60 e-folds of inflation. To see the maximum appearing, we can take the large $x$ limit of the potential

$$
\lim _{x \rightarrow \infty}\left(\frac{32 \pi^{2}}{\mu^{4}}\right) V_{\mathrm{CW}}=3+4 \ln (x)+2 y^{2} x^{2}(1+4 \ln (x))
$$

The slope of the potential at large $x$ gets a positive contribution from the $y^{0}$-term, and a positive or negative contribution from the $y^{2}$ correction; if the latter is negative, the potential has a maximum. The slope is

$$
\lim _{x \rightarrow \infty} \partial_{x} V_{\mathrm{CW}}=\frac{4}{x}+4 x y^{2}(3+4 \log (x))
$$

which vanishes for

$$
x_{\max }^{2}=-\left(y^{2}\left(3+4 \ln \left(x_{\max }\right)\right)^{-1}\right.
$$

which is only a solution for $y^{2}<0$. Numerically we find for $\kappa_{S H}=\mathcal{O}(1)$ that $x_{\max }=50-100$ in the absence of moduli corrections (i.e. using (2.6)).

\section{References}

[1] S. Antusch, K. Dutta and P. M. Kostka, Phys. Lett. B 677 (2009) 221 [arXiv:0902.2934 [hep-ph]].

[2] A. D. Linde, Phys. Rev. D 49 (1994) 748 [arXiv:astro-ph/9307002].

[3] A. D. Linde and A. Riotto, Phys. Rev. D 56, (1997) 1841 [arXiv:hep-ph/9703209].

[4] T. Banks, M. Berkooz, S. H. Shenker, G. W. Moore and P. J. Steinhardt, Phys. Rev. D 52 (1995) 3548 [arXiv:hep-th/9503114].

[5] J. P. Hsu, R. Kallosh and S. Prokushkin, JCAP 0312 (2003) 009 [arXiv:hep-th/0311077].

[6] E. J. Copeland, A. R. Liddle, D. H. Lyth, E. D. Stewart and D. Wands, Phys. Rev. D 49 (1994) 6410 [arXiv:astro-ph/9401011].

[7] M. Dine, L. Randall and S. D. Thomas, Phys. Rev. Lett. 75 (1995) 398 [arXiv:hep-ph/9503303].

[8] S. Kachru, R. Kallosh, A. D. Linde and S. P. Trivedi, Phys. Rev. D 68 (2003) 046005 [arXiv:hep-th/0301240].

[9] J. J. Blanco-Pillado et al., JHEP 0411 (2004) 063 [arXiv:hep-th/0406230].

[10] J. J. Blanco-Pillado et al., JHEP 0609 (2006) 002 [arXiv:hep-th/0603129].

[11] J. P. Conlon and F. Quevedo, JHEP 0601, 146 (2006) [arXiv:hep-th/0509012].

[12] M. Cicoli, C. P. Burgess and F. Quevedo, JCAP 0903, 013 (2009) [arXiv:0808.0691 [hep-th]].

[13] G. R. Dvali, Q. Shafi and R. K. Schaefer, Phys. Rev. Lett. 73 (1994) 1886 [arXiv:hep-ph/9406319].

[14] P. Brax, C. van de Bruck, A. C. Davis and S. C. Davis, JCAP 0609 (2006) 012 [arXiv:hep-th/0606140]. 
[15] Ph. Brax, C. van de Bruck, A. C. Davis, S. C. Davis, R. Jeannerot and M. Postma, JCAP 0701 (2007) 026 [arXiv:hep-th/0610195].

[16] S. C. Davis and M. Postma, JCAP 0804 (2008) 022 [arXiv:0801.2116 [hep-th]].

[17] R. Kallosh and A. D. Linde, JHEP 0412 (2004) 004 [arXiv:hep-th/0411011].

[18] R. Kallosh and A. D. Linde, JHEP 0702 (2007) 002 [arXiv:hep-th/0611183].

[19] J. P. Conlon, R. Kallosh, A. D. Linde and F. Quevedo, JCAP 0809 (2008) 011 [arXiv:0806.0809 [hep-th]].

[20] S. C. Davis and M. Postma, JCAP 0803, 015 (2008) [arXiv:0801.4696 [hep-ph]].

[21] H. Y. Chen, L. Y. Hung and G. Shiu, JHEP 0903 (2009) 083 [arXiv:0901.0267 [hep-th]].

[22] M. Badziak and M. Olechowski, arXiv:0911.1213 [hep-th].

[23] M. Badziak and M. Olechowski, JCAP 0902 (2009) 010 [arXiv:0810.4251 [hep-th]].

[24] M. Badziak and M. Olechowski, JCAP 0807 (2008) 021 [arXiv:0802.1014 [hep-th]].

[25] S. Antusch, M. Bastero-Gil, S. F. King and Q. Shafi, Phys. Rev. D 71 (2005) 083519 [arXiv:hep-ph/0411298].

[26] S. R. Coleman and E. J. Weinberg, Phys. Rev. D 7 (1973) 1888.

[27] E. Komatsu et al. [WMAP Collaboration], Astrophys. J. Suppl. 180 (2009) 330 [arXiv:0803.0547 [astro-ph]].

[28] K. Becker, M. Becker, M. Haack and J. Louis, JHEP 0206 (2002) 060 [arXiv:hep-th/0204254].

[29] M. Berg, M. Haack and E. Pajer, JHEP 0709, 031 (2007) [arXiv:0704.0737 [hep-th]].

[30] M. Cicoli, J. P. Conlon and F. Quevedo, JHEP 0801, 052 (2008) [arXiv:0708.1873 [hep-th]]. 